\title{
Do Mountains Exist? Towards an Ontology of Landforms
}

\author{
Barry Smith and David M. Mark \\ Departments of Philosophy (Smith) and Geography (Mark) \\ National Center for Geographic Information and Analysis, and Center for \\ Cognitive Science \\ University at Buffalo \\ Buffalo, New York 14261 U.S.A. \\ phismith@buffalo.edu,dmark@geog.buffalo.edu \\ Preprint version of a paper published in \\ Environment \& Planning B (Planning and Design), 30(3) (2003), 411-427
}

\begin{abstract}
The paper begins with the question "Do mountains exist?" It shows that providing an answer to this question is surprisingly difficult, and that the answer which one gives depends on the context in which the question is posed. Mountains clearly exist as real correlates of everyday human thought and action, and they form the archetype for geographic objects. Yet individual mountains lack many of the properties that characterize bona fide objects, and mountains as a category also lack many of the properties that characterize natural kinds. In the context of scientific modeling of the environment, especially of such phenomena as surface hydrology and fluvial erosion and deposition, mountains are not picked out as constituents of reality in their own right at all; rather they are just parts of the field of elevations whose gradients shape the direction of runoff and influence the intensity of erosion. Thus while an object-based ontology of mountains and other landforms is required to do justice to our everyday conceptions of the environment, and to support spatial reasoning and natural language processing, topographic databases designed to support environmental modeling can be field-based at geographic scales.
\end{abstract}




\section{Introduction}

Do mountains exist? The answer to this question is surely: yes. In fact, 'mountain' is the example of a kind of geographic feature or thing that is most commonly cited by speakers of English (Mark et al., 1999; Smith and Mark 2001), and several other languages. But whether they are considered as individuals (tokens) or as kinds (types), mountains do not exist in quite the same sense as do such prototypical everyday objects as chairs or people, crows or cups.

The most typical examples of such everyday objects studied in cognitive science fall into two large categories. On the one hand are organisms, products of natural selection; on the other hand are artifacts, products of deliberate design. Typically, instances of organisms and artifacts are objects or things, bona fide wholes with determinate, prominent, and complete boundaries that enclose them and separate them from their environments. It is not an arbitrary matter, a product of mere fiat, where the boundaries of your cat end and those of your carpet begin. The psychologist J. J. Gibson, who developed the project of a so-called ecological psychology, referred to such things as detached objects. Not only are everyday things separated from each other by bona fide boundaries, something similar holds true also for the kinds (types, categories) to which such everyday things belong. These, too, commonly are separated from neighboring categories by categorial boundaries rather than continuous gradations. It is not an arbitrary matter where the natural kind cat ends and where neighboring kinds begin. The natural kinds cat and crow are separated from their neighbors by a long history of natural selection and evolution. Artifactual kinds such as cup and chair are separated from their neighboring kinds in similar fashion by the distinct functions with which they were associated by the people who designed and made them.

Although they are often named, and sometimes even worshipped, individual mountains do not satisfy the criteria for being objects which we have just outlined for organisms and artifacts. Mountains do not have determinate, prominent, and complete boundaries. While the boundaries between the mountain and the air above its upper slopes may be determinate, prominent, and crisp; it is usually the case that, as we proceed downwards towards the foot of the mountain, no single candidate boundary is distinguishable at all. And similarly in the order of kinds: the category mountain is not distinguished in bona fide fashion from neighboring categories such as hill, ridge, butte, plateau, plain, and so on. The kind mountain is not a product of natural selection, nor does it represent an artifactual kind with bona fide instances which have arisen as a reflection of special human intention or purpose. Indeed the kind mountain begins to seem more like such kinds as neighborhood or locality - as a kind that is demarcated, both as a type and in its tokens, as a mere reflection of human habits of perception and action (Montello et al., 2001). These habits of perception and action may, moreover, vary from one culture to another,so that what is called a 'Berg' in German may not coincide perfectly with what is called a 'mountain' in English. In this sense, the question "Do mountains exist?" is not quite as foolish as it may have earlier appeared. 
In this paper, we will expand upon these points and explain their implications for the geographic ontology that will be needed if information systems are to be built which are friendly to untrained users yet at the same time able to support environmental modeling and other GIS applications. Finally, we will address the question of the role of mountains as entities in scientific models of the environment.

\section{Ontology}

Ontology is the field that answers questions of being or existence (Simons 1987, Johansson 1989, Frank 1997). As a branch of philosophy, ontology studies the constituents of reality. An ontology of a given domain describes the constituents of reality within that domain in a systematic way, as well as the relations between these constituents and the relations of these to constituents of other domains. Terms such as 'domain', 'constituent', 'reality' and 'relation' are themselves ontological terms, as also are terms such as 'feature', 'object', 'entity', 'item', as well as 'being' and 'existence' themselves.

Different conceptions of existence have been favored by philosophers at different times in the history of the discipline, including conceptions according to which there are distinct levels or grades of existence (or different senses of term such as 'existence' or 'being'). In recent decades, however, a conception has come to predominate among philosophical ontologists according to which 'existence' is univocal. The basis of this conception is the predominance in contemporary philosophy of the language of the firstorder predicate logic as the preferred tool of formalization. Existence is then identified as that which is expressed by the existential quantifier of the predicate calculus, as for example in Quine's famous 'criterion of ontological commitment', according to which to be is to be the value of a bound variable in a true first-order formalization of a naturalscientific theory (Quine 1953). Atoms, electrons, cells, organisms, planets and solar systems exist, on this dispensation, and so also do sets and numbers and other abstract mathematical entities to the degree that quantification over the latter is required for the successful formulation of the theories of natural science. Lacks, shortfalls, absences, mere possibles, mythical creatures and fictional characters, on the other hand, do not exist (or as we should more properly say: the corresponding expressions do not refer).

We shall accept the univocal reading of 'existence' in what follows. This reading has one considerable advantage, namely that it allows us to answer two important questions about existence in one fell swoop. The first question is: do token entities (instances, individual examples) of a given kind or category $\mathrm{K}$ exist? The second question is: does the kind or category $\mathrm{K}$ itself exist? To answer both questions simultaneously, we need only to determine whether quantification over entities of kind $\mathrm{K}$ is needed in order to support scientific predictions of some relevant sort. In this way we can avoid all of the difficult issues (referred to by philosophers under the heading 'problem of universals') pertaining to the question whether kinds or categories themselves exist independently of the tokens or particulars which instantiate them (Armstrong 1978). 
A second reason for accepting the univocal reading of 'existence' is that, as we have argued (Smith and Mark 2001), this reading conforms very well with commonsensical uses of the term in those contexts where 'exists' is being used in its literal sense (as contrasted, for example, with contexts where we are discussing detective stories and we ask questions like: 'did there exist any clues as to the identity of the murderer?'). To say that entities of a given sort exist in the literal sense is to say that such entities are constituents of reality. Reality itself is then not confined to the reality of microphysical entities described in the theories of contemporary physics. For there are entities and patterns of regularity not just at the microphysical level but at many different orders of aggregation of matter on higher levels. There are thus many different kinds of existents, from quarks to planets and from nucleotides to vertebrates, and the taxonomies produced for the purposes of explanation at micro-physical levels of organization may cross-cut those produced in relation to the organization of entities at higher levels.

This being said, one way to formulate our question "do mountains exist?" is as follows: do we need to accept (refer to, quantify over) mountains in order to attain good explanations or, what comes to the same thing, good (predictive, scientific) theories? Inspection of such scientific disciplines as geomorphology or hydrology suggests that the answer to this question is: no.

On the other hand, however, if we want to construct a scientific theory of animal behavior, then reference to geospatial forms such as mountain or valley may be indispensable. An entomologist describing the spatial behavior of ladybird beetles in California is aided in making predictions by the availability of the concept of a mountain when he finds it necessary to talk about the ways in which beetles concentrate together spatially (on mountain tops) in certain seasons (Edwards, 1957). This is so even though the beetles themselves might be strictly speaking in complete ignorance of mountains or other landforms; at certain times of year they just go up gradients, and in some landscapes such uphill travel takes them to mountain tops. (Compare the way in which $E$. coli bacteria go up sugar-density gradients, and so seem to be seeking out sugar.)

Appeal to the concept of mountain can help us, too, in understanding and in making predictions about human behavior. The role played by mountains in determining differential patterns of human settlements is analogous to the role played by islands in giving rise to the differential patterns of plant and animal evolution studied in island biogeography (MacArthur and Wilson, 1967). This and related disciplines thus provide evidence against a radical view to the effect that mountains would be mere façons de parler - or in other words that they would be tantamount to humanly constructed fictions, analogous to devils or spirits. The disciplines in question rather point to the possibility that mountains and other instances of landform categories do indeed exist, but at the interface between the perception and behavior of organisms on the one hand and the large-scale physical environment on the other. We note in passing that in this they are similar to those entities referred to by Gibson as affordances: "The affordances of the environment are what it offers the animal, what it provides or furnishes, either for good or ill" (Gibson, 1986, p. 127). "A path affords pedestrian locomotion from one place to 
another" (Gibson, p. 36). "A brink, the edge of a cliff, ... is a falling-off place. It affords injury and therefore needs to be perceived by a pedestrian animal" (Gibson, p. 36).

It is important to stress that affordances for Gibson-including those affordances called hills and mountains - exist as part of the perceived environment. Thus it is not merely because people have concepts of mountains and similar features that it is necessary to appeal to landforms in giving an account of their beliefs and behavior but also because such concepts relate to corresponding parts of their environments in direct and specific ways. Mountains, in this sense, do really exist. The concept of mountain is not like that of devil or ghost. For while beliefs involving the latter concepts do indeed effect some human behavior, they do so indirectly, just as the concept mountain is involved only indirectly in some religious and mythological contexts; for example, an anthropological account of Navajo beliefs about human origins would not be possible if the anthropologist could not refer to the four sacred mountains that represent the cardinal directions in the Navajo cosmography (Bingham and Bingham, 1982).

\section{Ontology and Information Systems}

People in general engage with the world from day to day in a variety of different ways: in addition to acting in the world directly, we, in our cultures, use maps, read newspapers, and listen to weather forecasts and traffic reports. Each of these ways of engaging with

the world involves a certain conceptualization, which means that it involves a system of concepts or categories which divide up the pertinent domain into objects, qualities, relations, and so forth.

The term 'ontology' is nowadays used by information scientists, in a sense distinct from that promulgated by philosophers, to designate the regimentation of such conceptualizations through the development of tools designed to render them explicit (Guarino 1998, Welty and Smith 2001, Smith 2002). Ontology in this sense is not concerned with questions of existence. It is designed rather to assist in the task of specifying and clarifying the concepts employed in given domains, above all by formalizing them within the framework of some formal theory with a well-understood logical (syntactic and semantic) structure. Since existence is not an issue, information systems ontologists can use their methods to examine conceptualizations from a much wider variety of domains. They can for example examine concepts such as God, salvation or sin used in a religious ritual setting, or concepts such as magic spell, wizard. or witch used in a story-telling setting, and so on. Such concepts refer to objects conceived as mere 'posits,' or 'models,' independently of any counterparts such posits might have in some independent reality.

Gruber (1993) defines an ontology in the information systems sense as "a specification of a conceptualization". He proposed this definition as part of the attempt to address the serious problems which arise in the field of information systems as a result of the fact that different groups of data-gatherers use different and often idiosyncratic terms and concepts in their work. One central goal of ontological regimentation is the resolution of 
the incompatibilities which result in such circumstances, ideally through the provision of some sort of generally accepted formal framework, a 'concise and unambiguous description of principal, relevant entities of an application domain and their potential relations to each other' (Schulze-Kremer 1997, p. 273). The hope is that, when once such a concise and unambiguous description, some central master ontology, has been established, then the different conceptualizations of different communities of information users could be calibrated in terms of this single canonical description in such a way that they would all be automatically intertranslatable.

The value of ontology in the information systems sense turns on the need to facilitate reusability of data and information gathered on the basis of different conceptualizations (for example data about air pressure at specific points in space, on the one hand, and data about weather patterns across large regions of space, on the other hand). It is designed to provide computationally tractable, robust, neutral frameworks within which data deriving from different sources can be rendered intercommunicable

It cannot be stressed too much that from the information science perspective, ontology is a strictly pragmatic enterprise. Returning to our present case, ontology in the information systems sense is not concerned with questions such as "Do mountains exist?" Rather, it starts with the conceptualizations that potential users of a given information system already have about the world, and goes from there to a description of corresponding domains of (real or putative) objects, all of which would be conceived, again, as mere 'posits' or 'models'. This variety of ontology is concerned with questions such as: "Should mountain be a category in our knowledge base?" "If so, how should the category mountain be defined?" and "How should individual mountains be represented in a database?"

\section{Primary Theory}

To see how we can merge the philosophical and information systems approaches to ontology within a single framework, let us return to our discussion, above, of the role played by the concept mountain in biological explanations. We argued that mountains and other landforms, if they exist at all, are to be located at the interface between the behavior of organisms on the one hand and the large-scale physical environment on the other. Thus we need to say more about the world in which humans and animals live. In this section, we concentrate more specifically on the world of everyday human activities. We can think of the latter, provisionally, as being just the world that is described by standard physics. But while physicists might focus upon objects visible at microscopic or macroscopic resolutions, the world of our everyday activities comprehends those objects which are set into relief when we use a resolution somewhere between these two extremes (Bittner and Smith 2001). Our everyday activities relate to mesoscopic phenomena in the realm that is immediately accessible to perception and action, they pertain to phenomena such as tables and boats, tabletops and snow, neighborhoods and streets. 
This is, once again, the realm of affordances in Gibson's terms, and it is important in this connection to emphasize that Gibson embraced a realist doctrine in his psychological theorizing. This means that he held that affordances exist in the environments of those organisms that relate to them in their actions. If we are to do justice to Gibson's insights, therefore, we must make a distinction between two sorts of conceptualizations: on the one hand are those conceptualizations that can be interpreted in realist fashion as relating to independently existing entities in reality, on the other hand there are those conceptualizations (arising for example in the realms of fiction or myth) which cannot be so interpreted. It is by exploiting this distinction between two different types of conceptualizations that we are able to merge the philosophical and information systems approaches to ontology within a single framework.

In support of the dichotomy between these two sorts of conceptualizations we can employ the distinction drawn by the anthropologist Robin Horton between what he called 'primary' and 'secondary' theory. Primary theory, for Horton (1982), is that part of common sense which we find in all cultures and in all human beings. 'Secondary theories', in contrast, are those collections of beliefs - folk or scientific-which are peculiar to particular social settings. Primary theory consists of basic (naïve or commonsense) physics, basic (folk or 'rational') psychology, and other families of those basic theoretical beliefs which all people need in order to perceive and act in ordinary everyday situations (Forguson 1989). Importantly, primary theory will be in many respects incomplete. Thus while primary theory recognizes weather phenomena, it has no explanation as to how such phenomena arise. While primary theory acknowledges the existence of mountains, it has no explanation of how mountains are demarcated (or how they fail to be demarcated) from their surrounding foothills and from the Earth beneath them.

Secondary theory, in contrast, consists both of scientific theories of microscopic and macroscopic physical phenomena such as atoms and quarks, galaxies and quasars, and also of folk beliefs relating to germs and evil spirits, heaven and hell, gods and devilsphenomena which are not accessible in our everyday perception and action because they are too large or too small, or perhaps because they do not exist at all. Primary theory is, as Horton points out, developed to different degrees by different peoples in its coverage of different areas - a primary theory of snow, for example, may be absent or underdeveloped in some isolated tropical climates, a primary theory of mountains may be absent or underdeveloped in isolated flatland communities, etc. In other respects, however, above all in its structure and in its basic orientation around objects and phenomena of human scale, primary theory differs hardly at all from culture to culture. In the case of secondary or 'constructive' theory, in contrast, differences of emphasis and degree give way to startling differences in kind as one moves from community to community and from culture to culture. For example, a Western anthropologist brought up with a purely mechanistic view of the world may find the spiritualistic world-view of an African community alien in the extreme (Horton, 1982, p. 228). 
There is a high degree of cross-cultural agreement in primary theory, and also a high degree of correspondence between primary-theoretical beliefs and the reality towards which they are directed. Both of these features of primary theory have evolutionary roots: there is a sense in which our shared beliefs about mesoscopic physics must, with a very high degree of likelihood, correspond to the reality they purport to represent, for if they did not, we could scarcely have survived as a species. It is for this reason, too, that primary theory must be compatible with the results of science (Smith 1995a, 1995b). In general we can assume that primary theory will not directly conflict with scientific theories of the same phenomena at the same granularity. At the same time, this evolutionary aspect allows us to explain how the beliefs widely shared from culture to culture should have a predominant focus on those parts and features of mesoscopic reality that are relevant to our specific human aims and to the specific human organs and sensory modalities that we have available for achieving these aims. Primary theory in general is above all tailored to the specific kinds of senses and motor abilities that are characteristic of the human species and to the associated manual technology that has formed the main support of human life from the birth of the species down to the present day (Horton, 1982, p. 232).

From the perspective of survival, Horton argues, we can believe what we like concerning micro-spirits and macro-devils, atoms and galaxies, residing on levels above or below the levels of everyday concern. But as far as the broad physical structures of everyday reality are concerned we have been constrained to believe the truth-otherwise we would not be here. Already for this reason we can expect that the commonsensical world as the world that is apprehended in primary theory will be to a large degree universal. It is something that is apprehended in very similar ways in all cultures as embracing a plurality of enduring substances (especially people and artifacts) possessing sensible qualities and undergoing changes (events and processes) of various regular sorts. It is apprehended as existing independently of our knowledge and awareness and as constituting a single whole that is extended in space and time and that is connected together via harmonious and intelligible causal relations (Forguson 1989). This body of belief about general regularities in the mesoscopic domain is put to the test of constant use, and survives and flourishes in very many different environments. Our common-sense beliefs are readily translated from one language to another, and judgments expressing such beliefs are marked by a widespread unforced agreement.

\section{Categories}

Primary theory, we hypothesize, is organized to a very large degree in terms of objects falling under categories (such as dog, table, hand, or axe). Such categories, like all common-sense categories, are marked by the feature of prototypicality. This means that, as Rosch (1973, 1978), Keil (1979) and others have shown, for most such categories, some members are better examples of the category than others, and are cognized as such. That is to say, people can in normal circumstances distinguish easily between the prototypical instances at the core of common-sense categories and the fringe instances in the penumbra. Furthermore, for many such categories, there is a great degree of agreement among human subjects as to what constitute good and bad examples. For 
example, robins and sparrows are widely considered to be good examples of bird, whereas ostriches and penguins and even ducks are considered to be poor examples. As Barsalou has pointed out: "No other categorization phenomena is more widespread or fundamental than graded structure" (1992, p. 33).

Each family of common-sense categories is organized hierarchically in the form of a tree, with more general categories at the top and successively more specific categories appearing as we move down each of the various branches. Rosch (1978) distinguishes one special level within each such tree, which she called the 'basic level' of cognitive classification. This consists of categories such as duck, zebra, clock, and fork, which infants learn very easily while experiencing difficulty learning terms such as mammal or utensil. Basic-level categories represent a compromise in cognitive economy between two opposing goals: that of informativeness, on the one hand, and that of minimizing categories based on irrelevant distinctions, on the other. The basic level (chair, apple) falls between the superordinate level (furniture, fruit), which is in general insufficiently informative, and the subordinate level (lounge chair, golden delicious), which adds too little informativeness for its additional cognitive cost.

Associated with each family of naïve categories is a certain unified domain, analogous to the subject-matter of a scientific theory. Our common-sense categories are organized in groups, corresponding to what Murphy and Medin (1985) called theory-like structures. When we learn categories, we learn at the same time how the things falling under these categories are related to each other and how they interact causally. When we acquire the category bird, for example, we learn that birds (typically) have wings, that birds (typically) can fly, and that these two features are interrelated.

As Dowty (1998) expresses it, it is possible to distinguish a limited number of broad category-domains, including the domain of naïve physics (organized by the principle of causation), the domain of animals (organized by the principle of what can move itself), and including especially the domain of human beings (organized by the principle that actions are caused by beliefs and desires).

The domains in question reflect innate dispositions to focus upon certain features and dimensions of reality - and on certain sorts of connections and functional and causal associations - and to ignore or de-emphasize others. In acquiring a given concept, for example a concept in the domain of animals or artifacts, very young children attend only to certain kinds of attributes as potentially diagnostic for the concept and ignore other attributes as irrelevant. For example, animate objects that can move of their own accord are distinguished by children 6-11 months old (Dowty 1998). As Keil noted, in learning concepts, "people do not simply note feature frequencies and feature correlations; they have strong intuitions about which frequencies and correlations are reasonable ones to link together in larger structures and which are not" (Keil 1994, p. 170). Without such intuitions, people could not learn the corresponding concepts, since they would have no way to select from the potentially infinite number of feature correlations by which they are confronted in their day-to-day lives (Spelke 1990, Spelke and Hermer 1996). 


\section{The Primary Theory of the Geographic Domain}

We have distinguished a number of theory-like domains - of naïve physics, of living things, of human beings and of artifacts - within the wider domain of primary theory. One formulation of our question "Do mountains exist?" might now read as follows. Is there an analogous theory-like domain of geographic objects within primary theory, and are the categories for mountain and other landforms part of that domain? We have proposed exactly that in earlier papers (Smith and Mark, 1998; Mark et al., 1999; Smith and Mark, 2001), expanding on the idea of a 'naive geography' advanced by Egenhofer and Mark (1995) as relating to a domain parallel to that of naive physics but comprehending objects at larger spatial and temporal scales. Smith and Mark (2001) explicitly added the geographic domain to Dowty's list of the "limited number of broad category-domains" that underlie the primary theory of the common-sense world. We hypothesized that the child conceptualizes the geographic world as a large unitary background extending outward in all directions from his or her location, comprising those larger parts of the environment which do not move, and we hypothesized also that the child, early on, has learned to appreciate that there is a difference between things that move, whether by themselves or because caused to move by another object, and the environment within which things move and which allows him or her to get from place to place. We can go further — on the basis of considerable supporting data — and advance the hypothesis that the pertinent basic level categories in this geographic domain of primary theory are precisely entities such as mountain, hill, island, lake, and so forth (Mark et al., 1999; Smith and Mark, 2001). We know of no data on the ages at which young children acquire or master the basic concepts of naive geography and the associated kinds of objects, and this is an important topic for future research. However, the presence of common geographic kinds such as mountains, hills, lakes, and rivers in nursery rhymes and stories intended for very young children suggests that these concepts are understood at quite an early age. For example, there is ample data that well before the age of four years, children can navigate through complex spaces from memory, understand maps and aerial photos, and that they can help others with navigation (Hazen et al., 1978; Spencer and Blades, 1985; Freundschuh, 1990; Blaut, 1997; Blades et al., 1998). The domains addressed by such cognitive activities are populated by or composed of geographic entities.

\section{Objects vs. Fields}

Can we extend these conclusions, now, from the realm of primary theory to the realm of science? To answer this question we must draw attention to a peculiar characteristic of the realm of primary theory: it is to a large degree organized ontologically in terms of objects or things, which have qualities (of being warm or cold, raw or cooked) and which undergo processes (of being bent or kissed or thrown). Among scientific disciplines, in contrast, we must draw an opposition between two kinds of ontological orientation. For while there are scientific disciplines that employ an ontology based centrally on objects, there are others that are based rather on fields, that is, upon continuous functions whose arguments are positions in some spatial domain and whose valuables are attributes of, for example, temperature, density, or the strength and orientation of a magnetic field. 
Examples of object-based scientific disciplines include particle physics, molecular chemistry, cell biology, and human anatomy. Examples of field-based disciplines include quantum field theory, electromagnetism, hydrodynamics, and meteorology. We hypothesize that there is no parallel opposition in the realm of folk disciplines. The naive or folk disciplines appear to work exclusively (or at least overwhelmingly - wind and some other atmospheric phenomena may yield exceptions) with object-based representations of reality. This holds, too, in the realm of geospatial folk categories. Places, for example, are, like volcanoes and hurricanes, conceptualized by non-experts as objects, and the same is true even for the whole of space, which is conceptualized as the totality of places (perhaps as some sort of huge container).

The ontology of objects is itself, as we have seen organized on two levels: the level of individuals (tokens, particulars) and the level of kinds (categories, types, universals). Our cognition of individuals is often marked by our use of proper names (such as 'Mount Everest') and of indexical expressions (such as 'that hill'). Our cognition of kinds is marked by the use of common nouns such as 'mountain', 'plateau', 'ridge', etc.

As we noted already above, kinds or categories are organized hierarchically in a way analogous to the trees constructed for biological classification purposes, where lower nodes are called 'species' and upper nodes 'genera'. Here we use the term 'category' to refer indiscriminately to both the lower- and the higher-level nodes of all such trees, including nodes corresponding to basic-level categories in the sense of Rosch and also to top-level categories, of which the most important for our purposes is the category object itself.

\section{The Truth about Earth}

Each primary theory is a theory about what actually exists in reality-or more precisely in some part of reality that is relevant to human perception and action (and ultimately to human survival). In order to formulate a primary theory of the geographic world, it will therefore be necessary to provide first of all an inventory of background facts about the Earth that are beyond challenge. One important truth about Earth is that it is a roughly spherical planet with a rocky mantle and crust. (Of course, the spherical nature of the planet is not part of primary theory.) In more detail, planet Earth is somewhat flattened pole to pole into an oblate spheroid. Geodesists have defined a reference surface known as the geoid, which approximates the shape of the Earth and which provides a datum for measuring surface irregularities. And indeed there are slight irregularities in the elevation of the Earth's surface, slight with respect to the shape of the planet. Some 70 percent of the surface of Earth is covered by liquid water, and the planet is surrounded by a gaseous envelope called the atmosphere. A very thin layer of plants covers most parts of the land surface, and animals (including humans) move about among those plants. When the planet is viewed as a solid detached object, its surface would appear relatively smooth even if the water were removed. Variations above and below the geoid have a range of about $22 \mathrm{~km}$, and this is a very slight variation, about one third of one percent of the planet's radius of about $6400 \mathrm{~km}$. 
A complete ontology of geographic phenomena will have to incorporate all of these scientific facts and more, but only some of them are of relevance to the primary theory of the geographic domain. For when the Earth is viewed from a point upon its surface by a creature between one and two meters tall, it is precisely the aforementioned slight variations in the shape of the Earth's crust which dominate the landscape, while at the same time the curvature of the geoid, of the horizontal, is almost imperceptible. For now, therefore, we will ignore the curvature of the geoid, as in naïve geography (Egenhofer and Mark, 1995), and focus rather on those elevations or depths above and below the geoid which form the topographic environment for people (and indeed for all land-based organisms).

What this means, now, is that the primary theory of the geographic world is organized precisely around categories for major landforms such as mountain, hill, valley, island, etc., and for associated water bodies and watercourses such as lake and river. It is categories such as these that result when really existing variations in elevation (elevation fields), and the partial covering of lower parts of such surface are covered by water, and are subject to the object-based conceptualization that is imposed upon reality by primary theory. Primary theory seeks to make objects out of those variations in the geoid that are salient at a certain level of granularity. Or, as Gibson might have put it, primary theory seeks to make objects out of those variations in the geoid that afford particular human activities. It does this effectively by imposing fiat parsings upon the relevant field of elevations (Smith 2001). The results are (individual) mountains, hills, islands and so forth, and we can hypothesize that the relevant dimensions are parsed lexically (into mountains and hills, lakes and ponds, and so forth) in such a way as to effect a compromise between too many distinctions (which would be difficult to remember, and to apply effectively in situ) and too few (which would bring inefficiencies in communicating behaviorally relevant differences). Computational methods for extracting landform features from elevation fields have been developed to replicate the methods used by geomorphologists and others (Frank et al., 1986; Dikau, 1989; Usery, 1996; Dehn et al., 2001), and these may be pertinent to the task of understanding formally what is involved also in the fiat parsings effected by naïve subjects.

Primary theory recognizes not only objects but also corresponding attributes (properties, aspects, features) and it recognizes also the relations between objects. These form a secondary axis of the ontology, along with the attributes, events, processes, actions, states and the like in which such objects are involved. The system of objects forms the fundamental axis in this ontology in the sense that attributes are always attributes of objects, relations always relations between objects, events always events involving objects, and so forth. Thus primary theory recognizes that mountains have certain shapes, coverings, material constitutions; that some mountains are taller than, more difficult to climb than, other mountains, and so forth.

It is important to note that individual landforms are seldom bona fide objects with crisp boundaries of their own. The fiat parsings referred to above are marked by a high degree of vagueness or gradedness (Burrough and Frank 1996; Smith and Brogaard 2001) and of 
variability as between different cultures and perspectives (Dawson 1992). Landscapes themselves are formed by erosional and depositional processes that vary continuously over space in their intensity and effect. Where crisp slicings are instituted around the foothills of mountains, this will be as a result of politico-admistrative processes reflecting special conditions, for example pertaining to a need to license mining or tourism or to protect regions from trespass or invasion (Fisher and Wood, 1998).

When it comes to primary-theoretical categories it is noteworthy also that human beings are able to parse the landforms and waterforms they encounter by utilizing a remarkably parsimonious categorical repertoire, employing categories that are tuned to the types of regularity which have arisen as geological and other forces working from above and below have shaped the surface of the Earth in more or less intelligible ways. We have the capacity to assign instances to these categories, and this capacity extends even to those instances - such as coastal banks and icebergs - which undergo changes of shape and location. The surface of the Earth is, therefore, when viewed through the human conceptualization of primary theory, not sheer chaos; it is not a matter of kaleidoscopic randomness.

\section{Landscapes as Fields}

The influence of gravity is one dominant factor giving rise to this (relative) order: loose material tends to move away from high areas toward lower ones in a process generally termed erosion. Steeper slopes are less stable than gentle ones and so over time there is a tendency toward leveling. Overhanging cliffs are extremely rare - all of which implies also that, with almost no loss of generality, the elevation of the Earth's surface relative to the geoid can be modeled or conceptualized scientifically as a single-valued function of horizontal position, that is, as a continuous field. And, generally speaking, this is how earth scientists have modeled the geometry of the Earth's surface.

The fact that the Earth's surface can be represented by a single-valued function of horizontal position has meant that scientists seeking to model, for example, hydrology or sediment transport, are able to conceptualize the Earth's surface as composed of slope gradients and orientations over a field of elevations. This same fact has had implications also for scientific cartography. Maps of the type with which we are familiar are possible only because the surface of Earth itself approximates so closely to the state of twodimensionality. The first maps portraying topography using isolines called 'contours' appeared around 1800, and a mathematical theory of the topology of such continuous, smooth single-valued surfaces was presented more than a century ago in Cayley's 1859 paper "On contour lines and slope lines" and in Maxwell's 1870 extension of Cayley's work "On hills and dales". This idea of the single-valued field of elevations as a representation of the form of the Earth's surface has been incorporated implicitly or explicitly into representations of earth forms developed for computers since the 1950s.

As is reasonable, given that they are standardly designed to address a variety of goals, maps commonly embody elements of both the field-based view of the Earth's surface in terms of elevations (fields) and the object-based view embraced by primary theory. This 
becomes especially evident when we look at how mountains are represented on typical maps by means of contour lines, which are used to represent the shape of the Earth's surface, including the shapes of landforms, but not the landforms themselves. Cartographers often put the names of mountains on their maps, in the neighborhood of hill shading or of some corresponding parts of contours that indicate the shape of the Earth's surface in the neighborhood of the label. But then they leave it up to the user of the map to infer the extent of the object to which the name refers. Exactly what part of the map is Mount Washington? Which part of the Himalayas is Mount Everest. Maps do not represent mountains directly as objects with crisp boundaries - they rarely if ever show the boundaries of mountains at all. And this is in one sense correct: it captures an important feature of mountains as they exist in the reality that is grasped by primary theory, namely that they are objects whose boundaries are marked by gradedness or vagueness. In another sense, however, it has a misleading consequence. For, if it is suggested that what exists in the primary geographic realm is to be identified with what is represented in maps, then we would have to conclude, incorrectly, that mountains do not exist.

There is an interesting comparison to be made, in this connection, between mountains and neighborhoods. A neighborhood or region such as downtown Santa Barbara, too, is not represented on maps. At best one has a label, associated with the part of Santa Barbara accepted by the largest number of people as belonging to the downtown area; the boundaries of downtown Santa Barbara are not delineated at all. The reason for this is as follows. Downtown Santa Barbara, like your particular favorite mountain, is marked by gradedness as concerns the readiness of people to accept given regions as belonging or not belonging to it as parts (Montello et al., 2001). The central parts of downtown Santa Barbara are like the highest parts of your favorite mountain in that they are also the least problematic parts. We confess that this similarity between mountains and neighborhoods implies a certain paradox. For while many would be happy to accept that downtown Santa Barbara is in some sense a product of socially established habits and beliefs, and recognize that downtown Santa Barbara would not exist in the absence of such habits and beliefs, there are few who would be similarly happy to accept that Mount Everest exists only because there are corresponding cognitive or behavioral practices on the part of human beings. Surely, we want to say, Mount Everest, like the planet as a whole, was there long before large mammals such as ourselves entered the scene and began to think and act in relation to our environment.

The paradox can, however, be resolved: every molecule of Mount Everest exists, and is located exactly where it is located, completely independently of our human beliefs. (The same applies, too, to every molecule of downtown Santa Barbara.) What depend on human beliefs are, rather, certain speech practices: we call the land up to here 'Mount Everest', we do not call the land down there 'Mount Everest'. Mount Everest is then the result of projecting these speech practices onto the surface of the Earth in such a way that they mark out a certain territory with a certain shape and material constitution. This territory, with exactly this shape and material constitution, was there many millennia before humans came along. To put the point another way (and ignoring, for the moment, 
the issue of vagueness): this particular portion of geophysical reality which we call 'Mount Everest' has long existed independently of all human cognitive practices. But so also did many other, only slightly different portions of geophysical reality, none of which acquired a name or were in any other sense cognitively set into relief from their surroundings. As an object, therefore, which was delineated, marked out, demarcated, set into relief in this fashion, and thereby also named, Mount Everest exists only as a result of human beliefs and habits. In this sense Mount Everest is, like downtown Santa Barbara - and like professions, religions, human ethnic groups and other similar phenomena (Smith 1999)- a product of socially established beliefs and habits. ${ }^{1}$ It is a fiat object. As a portion of geophysical reality, in contrast, which is to say: a certain large aggregate of molecules connected together in space in just this fashion, Mount Everest exists entirely independently and had already existed for a long period of time before we developed out current cognitive abilities.

One residual problem remains, however, which turns on the vagueness of mountains and neighborhoods. What, precisely, do we mean by the territory that is delineated by our use of the name "Mount Everest" (Varzi, 2001)? This is, unfortunately, a difficult problem, one which we will not attempt to resolve here.

\section{Geographic Objects}

The topographic environment, as experienced by people and other mobile organisms of roughly human size, is very different from the single-valued surface described by those Earth scientists who employ field-based conceptualizations. Of course it is the same environment in both cases - this is the core of Gibson's realism about affordances - but where in one case it is experienced through our human senses in the context of our human activities and needs, which means with a specific sort of granularity, which filters out irrelevant detail, in the other case it is experienced, quantitatively, via processes of measurement. When ordinary people see, learn, and describe a landscape, it seems that they do not think of the landscape as a field in the scientific sense of a surface of elevations. Instead they parse it into objects, presumably based on some combination of the application of Gestalt principles governing visual perception and on the recognition of affordances. Visual perception tends to identify convex surfaces as enclosing objects or potential objects. Gibson, as we saw, distinguished detached objects, which have completely closed surfaces, making them moveable, at least in principle, and attached objects, the latter projecting out of, or forming parts of the surface layers of, larger objects (the nose in your face, the handle on your door). The perceived surface of the Earth appears to be populated by those very large attached objects that we call landforms. Convex parts are most salient, although concave regions (holes) such as valleys and

1 Interestingly, John Searle, whose 1995 book is thus far the most sophisticated treatment of the ontology of social reality, uses Mount Everest as his preferred example of an object belonging to the realm of brute facts, i.e. of objects which are independent of all human beliefs and institutions. See Searle, 1995, pp. 41, 61, 153 et passim. 
craters also appear to be seen as objects in many contexts. In an experiment described by Smith and Mark (2001), subjects were asked to list examples of geographic features, objects, or things. In every case mountain was the most frequently listed example. Evidently, mountains are the quintessential geographic things to people in everyday contexts, yet they hardly appear in the scientific models. Nor do they appear as objects in our geographic databases. Mountains and the like have also been neglected in philosophers' ontologies, which have taken as their paradigm for objects Gibsonian detached entities, i.e. complete, moveable things with their own boundaries, such as organisms, atoms, or planets.

\section{Water Forms}

We mentioned gravity as one central force tending to lead to a certain intelligibility (simplicity) of the Earth's surface, both when viewed from a scientific perspective (as a field of elevations) and also when viewed from the naïve perspective of human primary theory. Another such central force has to do with hydrology. Water covers some 70 percent of the Earth's surface, and the presence of liquid water at the surface is a special characteristic of the planet. Most of the water is collected into a single connected water body known as the oceans (or the sea). But some water evaporates from this surface, is temporarily held and transported by the atmosphere, and falls again as precipitation. When it falls on the land, it may infiltrate into the ground, or it may be retained locally and re-evaporate. However, much of the precipitation that falls on land eventually collects in relatively concave parts of the surface to form water bodies such as lakes and ponds, and watercourses such as rivers. Watercourses commonly move sediment as well as water, contributing to surface erosion in some areas and depositing surface materials in others. As Gibson has noted, water enjoys a quite special importance as forming the very environment of fish and other aquatic organisms. But water is also a very special component of the environment for most terrestrial animals, not only satisfying one central requirement for life itself, but also, when gathered at the surface in sufficient depth, forming a barrier to movement for creatures such as people.

\section{Topography and Environmental Modeling}

For all that has been said in the above about the predominance of object-based conceptualizations in the realm of primary theory, the examination of the scientific goals of environmental modelers tells us that they can still safely continue to focus on field representations of topographic elevation in their work, since the characteristics of environmental modeling are best explained as field-based, rather than object-based, schemata. For example, hydrologic phenomena vary greatly in scale, and also in the level of aggregation that underlies modeling. Surficial processes such as soil creep or overland flow are essentially two-dimensional, but groundwater modeling requires three spatial dimensions. Some models deal with steady states or equilibria, but others require high temporal resolution. As Mark (1987) pointed out, the field of drainage directions across a landscape can be conceptualized as a network. Surface runoff models include parameters such as infiltration rates and surface roughness. Models of hillslope erosion include 
several distinct processes, and are coupled to fluvial erosion and deposition in channels. These examples show the suitability of field representations for work in topography.

In contrast to some of the other application domains of environmental modeling and GIS, the main data infrastructure for hydrology, namely surface topographic or elevation data, has been a focus of attention in computer mapping and GIS since its earliest days. Early efforts to represent topography on computers attempted to use digitized contours, but computing about surfaces from contours turned out to be inefficient, and regular grids, lattices, and altitude matrices proved more effective (Boehm, 1967; Mark 1979). Later, it became popular to represent surfaces within GISs as sets of non-overlapping triangles in Triangulated Irregular Networks (TINs; see Mark, 1997, for a history of TINs). A formal ontology of elevation surfaces and of fluvial processes will be related to the data models used to represent topography in current GISs and is expected to reveal how fluvial process modeling can be implemented in the various representations.

Of course, some environmental models deal with individual plants, animals, and pieces of sediment. Already for this reason, therefore, a complete suite of tools for environmental modelers must include objects and movement of objects as well as fields representing environmental gradients. But also for reasons of enhancing usability, those engaged in the construction of environmental models must take account of the fact that much of what is dealt with in terms of object-based fiat parsings by the primary theory of the users of such models, including landforms, are treated in terms of fields in the models themselves.

\section{Ontology of Land Forms and the Status of Geomorphology}

Topography, insofar as it serves as input to environmental models, will continue to be modeled by field-like representations. This raises an interesting academic question: How does geomorphology, the science that purports to have landforms as its object of study, fit in here? Recently, the geomorphologist Bruce Rhoads (1999, p. 766, (emphasis added)), stated that "the extent to which geomorphology as a distinct field of science can be justified on ontological or epistemological grounds seems to depend on the extent to which landforms can be viewed as natural kinds." The term "natural kind' has been defined by Sankey (1997, p. 239), in keeping with standard work in the philosophy of science, as follows: "The objective reality investigated by science is populated by mindindependent natural kinds of things, which are characterized as such by the fundamental, intrinsic causal powers which they possess." Examples of natural kinds in this sense range from biological species, to the elements in the periodic table, to the six different kinds of quarks (up, down, charm, strange, top, and bottom). It follows from our arguments above that landforms are not natural kinds. Landform kinds do indeed exist; but their ontological status is of a secondary sort, to be ranked (perhaps) with kinds of settlement or feeding patterns among human populations, rather than with the very joints of reality. Does it follow from this that geomorphologists need to worry about the status of their discipline as a science?

Half a century ago, landforms as objects did indeed form the core of a geomorphological science. This is shown already by the three Greek roots from which the term 
geomorphology is derived, which together refer to: a discourse or science of earth forms (Thornbury, 1954, p. 1). But things have changed. We claim that Rhoads (1999) was mistaken in his comment to the effect that the scientific status of geomorphology depends on the admissibility of landforms to the pantheon of natural kinds as the latter are defined above. Even if landform kinds such as mountain, drumlin, delta, esker, gorge, cinder cone did not exist at all because the place of the corresponding tokens was taken by a chaotically irregular rocky crust of the planet Earth, we believe that geomorphology would still continue unscathed, for reasons that Rhoads himself pointed out in the introduction to the book The Scientific Nature of Geomorphology, in which he and Colin Thorn described "the emergence and rise to dominance of the process-oriented approach to geomorphology over the last 40 years" (Rhoads and Thorn, 1996). Since contemporary geomorphology is almost entirely concerned with understanding the processes that shape the Earth's surface, and with the question of how local elevations and slopes control the spatial distribution of those processes and their impacts, landforms-as-objects are in practice irrelevant to most subfields of geomorphology. Rhoads however may be correct to this extent: that much of the extra-scientific interest in geomorphology and its subfields derives from the possibility of expressing the results of its analyses of process in the non-scientific, primary-theoretic terminology of hills and dales, mountains and lakes.

\section{Conclusions}

A complete ontology of the geospatial world would need to comprehend not only the common-sense world of primary theory but also the field-based ontologies that are used to model runoff and erosion. The completion of such an ontology is a challenging task, involving serious research challenges ranging from ontological specification to algorithm development. But its realization would bring significant benefits, since many serious professional users of geographic information, such as pilots, soldiers, and scientists, hikers, wildfire fighters, and naturalists, must communicate about particular parts of the landscape as if they were objects, while at the same time drawing on the resources of field-based topographic databases.

\section{Acknowledgements}

This paper is based upon work supported by the National Science Foundation, Geography and Regional Science program, under Grant No. BCS-9975557. Support of the National Science Foundation is gratefully acknowledged.

\section{References}

Armstrong, David M. 1978 Universals and Scientific Realism, volume 1: Nominalism and Realism, volume 2: A Theory of Universals, Cambridge: Cambridge University Press.

Barsalou, Lawrence W. 1992. Cognitive Psychology: An Overview for Cognitive Scientists. Hillsdale, New Jersey: Lawrence Erlbaum Associates 
Bingham, Sam and Janet Bingham, eds. 1982. Between Sacred Mountains: Navajo Stories and Lesson From the Land. Tucson: University of Arizona Press.

Bittner, T. and Smith, B. 2001. A Taxonomy of Granular Partitions. In Spatial Information Theory. Proceedings COSIT '01, Lecture Notes in Computer Science, Berlin/New York: Springer.

Blades, M., Blaut, J., Darvizeh, Z., Elguea, D., Soni, S., Sowden, D., Stea, D., Surajpaul, R., and Uttal, D. 1998 A Cross-Cultural Study of Young Children's Mapping Abilities, Transactions of the Institute of British Geographers, 23: 2, 269-277.

Blaut, J. M. 1997 The Mapping Abilities of Young Children - Children Can, Annals of the Association of American Geographers, 87: 1, 152-158.

Boehm, B. W., 1967. Tabular Representation of Multivariate Functions with Application to Topographic Modeling. Proceedings, 22nd National Conference, Association for Computing Machinery, pp. 403-415.

Burrough, P. A. and Frank, A. U. (eds.) 1996. Geographic Objects with Indeterminate Boundaries, London and Bristol, PA: Taylor and Francis.

Cayley, A., 1859, On contour lines and slope lines. Philosophical Magazine, v. 18, pp. 264-268.

Dawson, A. 1992 The Relative Hills of Britain, Milnthorpe, Cumbria: Cicerone Press.

Dehn, M., Gärtner, H., and Dikau, R., 2001, Principles of Semantic Modeling of Landform Structures. Computers \& Geosciences 27, 1005-1010.

Dikau, R., 1989. The Application of a Digital Relief Model to Landform Analysis in Geomorphology. In: Raper, J. (Ed.), International Journal of Geographical Information Science Three Dimensional Applications in Geographical Information Systems. Taylor \& Francis, London, pp. 51-77.

Dowty, D. 1998 On the Origin of Thematic Roles Types. Invited lecture, Lexicon in Focus Conference, Wuppertal, August 1998. See http://www.ling.ohiostate.edu/ dowty/where-do-roles.abstract.

Edwards, J. G. 1957. Entomology Above Treeline: The Attraction of Ladybird Beetles to Mountain Tops. Coleopterists' Bulletin 11: 41-46

Egenhofer, M. J., and Mark, D. M., 1995. Naive Geography. In Frank, A. U. and Kuhn, W., editors, Spatial Information Theory: A Theoretical Basis for GIS, Berlin: Springer-Verlag, Lecture Notes in Computer Sciences No. 988, pp. 1-15.

Fisher, P. F., and Wood, J., 1998. What is a Mountain? or The Englishman who went up a Boolean Geographical concept but realised it was Fuzzy. Geography 83 (3), 247256

Forguson, Lynd 1989 Common Sense, London and New York: Routledge.

Frank, A. U. 1997 Spatial Ontology: A Geographical Point of View, in: O. Stock (ed.), Spatial and Temporal Reasoning, Dordrecht: Kluwer Academic Publishers, 135-153. 
Frank, A. U., Palmer, B., and Robinson, V., 1986. Formal Methods for Accurate Definition of Some Fundamental Terms in Physical Geography. In Proceedings, Second International Symposium on Spatial Data Handling, Williamsville, NY: International Geographical Union, Commission on Geographical Data Sensing and Processing,pp. 583-599.

Freundschuh, S. M. 1990 Can young children use maps to navigate? Cartographica, 27: 1, 54-66.

Gibson, J. J., 1986. The Ecological Approach to Visual Perception. Hillsdale, NJ: Lawrence Erlbaum.

Gruber T. R. 1993 A translation approach to portable ontologies. Knowledge Acquisition, 5(2):199-220.

Guarino, N. (ed.) 1998 Formal Ontology in Information Systems. Proceedings of FOIS'98, Trento, Italy, 6-8 June 1998, IOS Press, Amsterdam.

Hazen, N. L., Lockman, J. J., and Pick, H. L. 1978 The development of children's representation of large-scale environments, Child Development, 49, 623-636.

Horton, Robin 1982. Tradition and Modernity Revisited. in M. Hollis and S. Lukes (eds.), Rationality and Relativism, Oxford: Blackwell, 201-260.

Johansson, Ingvar 1989 Ontological Investigations. An Inquiry into the Categories of Nature, Man and Society, New York and London: Routledge.

Keil, F.C. 1979 Semantic and Conceptual Development: An Ontological Perspective, Cambridge MA: Harvard University Press.

Keil, F.C. 1994. Explanation Based Constraints on the Acquisition of Word Meaning. in L. Gleitman and B. Landau (eds.), The Acquisition of the Lexicon, Cambridge, MA: MIT Press, 1994, 169-196.

MacArthur, R. H., and Wilson, E. O., 1967. The Theory of Island Biogeography. Princeton, NJ: Princeton University Press.

Mark, D. M., 1979. Phenomenon-based data-structuring and digital terrain modelling. Geo-processing, 1, 27-36.

Mark, D. M., 1987. Network models in geomorphology. Chapter 4 in: M. G. Anderson, editor, Modelling Geomorphological Systems, New York: John Wiley and Sons, pp. 73-97.

Mark, D. M., 1997. The History of Geographic Information Systems: Invention and ReInvention of Triangulated Irregular Networks (TINS). Proceedings, GIS/LIS '97, Bethesda, MD: American Congress on Surveying and Mapping/American Society for Photogrammetry \& Remote Sensing, 284-289.

Mark, D. M., Smith, B., and Tversky, B., 1999. Ontology and Geographic Objects: An Empirical Study of Cognitive Categorization. In Freksa, C., and Mark, D. M., 
Editors, Spatial Information Theory: A Theoretical Basis for GIS, Berlin: SpringerVerlag, Lecture Notes in Computer Science No. 1661, pp. 283-298.

Maxwell, J. C., 1870, On hills and dales. Philosophical Magazine, v. 40, pp. 421-427.

Montello, D. R., Goodchild, M. F., Gottsegen, J, and Fohl, P., 2001. Things'll Be Great When You're Downtown: Behavioral Methods for Determining Referents of Vague Spatial Queries. In Cristani, M., and Bennett, B., (editors), Workshop on Spatial Vagueness, Uncertainty, and Granularity, CD proceedings, Leeds, UK: University of LeedsMurphy, Gregory L. and Medin, Douglas, 1985. The Role of Theories in Conceptual coherence. Psychological Review, 92: 3, 289-316.

Quine, W. V., 1953, On What There Is, as reprinted in From a Logical Point of View. New York: Harper \& Row.

Rhoads, B. L., 1999. Beyond pragmatism: The value of philosophical discourse for physical geography. Annals of the Association of American Geographers 89 (4), 760771 ,

Rhoads, B. L., and Thorn, C. E., 1996. The Scientific Nature of Geomorphology: Proceedings of the 27th Binghamton Symposium in Geomorphology. Chichester, New York : John Wiley \& Sons.

Rosch, E., 1973. On the internal structure of perceptual and semantic categories. In T. E. Moore (editor), Cognitive Development and the Acquisition of Language, New York, Academic Press, 111-144.

Rosch, E., 1978. Principles of categorization. In E. Rosch and B. B. Lloyd (editors) Cognition and Categorization. Hillsdale, NJ: Erlbaum, 27-48.

Sankey, H., 1997. Induction and Natural Kinds, Principia 1: 2, 239-254.

Schulze-Kremer, Steffen 1997. Adding Semantics to Genome Databases: Towards an Ontology for Molecular Biology. in Proceedings of the Fifth International Conference on Intelligent Systems for Molecular Biology, T. Gaasterland, et al. (eds.), Halkidiki, Greece, June 1997. Palo Alto: AAAI Press, 272-275.

Searle, John R., 1995. The Construction of Social Reality, New York: Free Press.

Simons, Peter M. 1987 Parts. An Essay in Ontology, Oxford: Clarendon Press.

Smith, B. 1995a Formal Ontology, Common Sense, and Cognitive Science. International Journal of Human-Computer Studies, 43, 641-667.

Smith, B. 1995b The Structures of the Commonsense World. Acta Philosophica Fennica, $58,290-317$.

Smith, B. 1999 Agglomerations, in C. Freksa, and David M. Mark, eds., Spatial Information Theory. Cognitive and Computational Foundations of Geographic Information Science (Springer Lecture Notes in Computer Science 1661), 267-282.

Smith, B. 2001 Fiat Objects. Topoi, 20: Number 2. 
Smith, Barry, 2003. Ontology: Philosophical and Computational. in Luciano Floridi (ed.), The Blackwell Guide to the Philosophy of Computing and Information, Oxford: Blackwell, in press.

Smith, B. and Brogaard, B. 2003 A Unified Theory of Truth and Reference, Loqique et Analyse, 43.

Smith, B., and Mark, D. M., 1998. Ontology and Geographic Kinds. in T. K. Poiker and N. Chrisman (eds.), Proceedings. 8th International Symposium on Spatial Data Handling (SDH'98), Vancouver: International Geographical Union, 1998, 308-320.

Smith, B., and Mark, D. M., 2001. Geographic categories: An ontological investigation. International Journal of Geographic Information Science, 15 (7), 591-612.

Spelke, E. S. 1990 Principles of Object Perception, Cognitive Science, 14: 29-56.

Spelke, E. S. and Hermer, L. 1996 Early Cognitive Development: Objects and Space, Perceptual and Cognitive Development. Ed. Rochel Gelman, Terry Kit-Fong, et al. San Diego: Academic Press. 71-114.

Spencer, C. and Blades, M. 1985 How children navigate, Journal of Navigation, 38: 445453.

Thornbury, William D., 1954. Principles of Geomorphology. New York: Wiley and Sons.

Usery, E.L., 1996. A Feature-Based Geographic Information System Model, Photogrammetric Engineering and Remote Sensing, Vol. 62, No. 7, pp. 833-838.

Varzi, A. C., 2001. Vagueness in Geography. Philosophy \& Geography, 4:1, 49-65.

Welty, C. and Smith, B. (eds.) 2001 Formal Ontology and Information Systems, New York: ACM Press. 